\title{
Active Neutral Particle Diagnostics on LHD by Locally Enhanced Charge Exchange on an Impurity Pellet Ablation Cloud
}

\author{
P.R. Goncharov ${ }^{1}$, T. Ozaki ${ }^{1}$, E.A. Veshchev ${ }^{2}$, V.Yu. Sergeev ${ }^{3}$, S. Sudo ${ }^{1}$, N. Tamura ${ }^{1}$, \\ D.V. Kalinina ${ }^{1}$, TESPEL Group ${ }^{1}$ and LHD Experimental Group ${ }^{1}$ \\ 1) National Institute for Fusion Science, Toki, Gifu 509-5292, Japan \\ 2) Graduate University for Advanced Studies, Hayama, Kanagawa, 240-0193, Japan \\ 3) St.Petersburg Polytechnical University, St.Petersburg, 195251, Russia
}

\section{Introduction}

Production, confinement and thermalization of high-energy particles are the fundamental issues in fusion plasma ion kinetics. The ion distribution function $f_{i}(v, v, t)$ and its evolution under the ion cyclotron heating $(\mathrm{ICH})$ and neutral beam injection (NBI) are studied by energy resolved charge exchange neutral particle flux measurements. For helical systems, such as LHD, local diagnostics are required due to the complex 3D magnetic configuration. First radially resolved measurements of local $\mathrm{H}^{0}$ atomic energy spectra have been made on LHD by the pellet charge exchange (PCX) method with a Compact Neutral Particle Analyzer (CNPA). The diagnostic technique, the initial measurement results in the range $10-100 \mathrm{keV}$ and the data analysis are described.

\section{Experimental Method}

An impurity cloud $\mathrm{r}_{\text {cloud }}<\mathrm{r}_{\text {plasma }}$ surrounding an ablating solid pellet provides a localized charge exchange target scanning the plasma radially. This is an advantageous approach to the local charge exchange neutral particle analysis [1] alternative to the use of a diagnostic neutral beam. The PCX atomic flux ${ }^{P C X}(E, \boldsymbol{r}(t))$ substantially exceeds the naturally occurring neutral flux and reflects the local plasma ion distribution function at the pellet position. Pellet-induced neutral fluxes were previously measured on LHD with a natural diamond detector [2]. However, obtaining the energy spectra from these data in the main energy range of interest $\left(10^{1}-10^{2} \mathrm{keV}\right.$ in the present experiments) is complicated due to the high operating speed, i.e. the spatial resolution requirement [3].

The new diagnostic presented here is based on a Compact Neutral Particle Analyzer (CNPA) [4] installed on LHD for measurements in the $\mathrm{H}_{0}$ energy range $1-170 \mathrm{keV}$. CNPA employs a thin $50 \AA$ diamond-like carbon stripping film instead of a traditional gas stripping cell to ionize the incoming neutrals, a strong permanent analysing magnet ( $~(1 \mathrm{~T})$ sorting the resultant protons by energies (Larmor radii) and an array of 40 channel electron multipliers (CEMs) for particle detection. The experiment is shown schematically in Fig.1. Pneumatically accelerated polystyrene $\left(-\mathrm{C}_{8} \mathrm{H}_{8^{-}}\right)_{n}$ balls are injected transversally; typical $\mathrm{D}_{p e l}=500-900 \mu \mathrm{m}$, $v_{p e l}=300-400 \mathrm{~m} / \mathrm{s}$ resulting in $1-2 \mathrm{~ms}$ pellet flight time to the full ablation in the core plasma. The angle between the analyzer sight line and the pellet injection axis is $2^{\circ}$ on the horizontal midplane projection and $1^{\circ}$ in the poloidal plane. The values of the local $v_{\|} / v$ for the observable particles are usually in the range -0.2 to +0.2 along the average pellet flight length.

The operating count rate $C$ in PCX experiments is dictated by the desired radial resolution $\delta l \lesssim 10^{-1} \mathrm{~m}$, the pellet velocity $v_{p e l}$ and the minimum statistically acceptable number of counts per one spectrum $N \approx 10^{3}$. Therefore, the analyzer should be able to process 


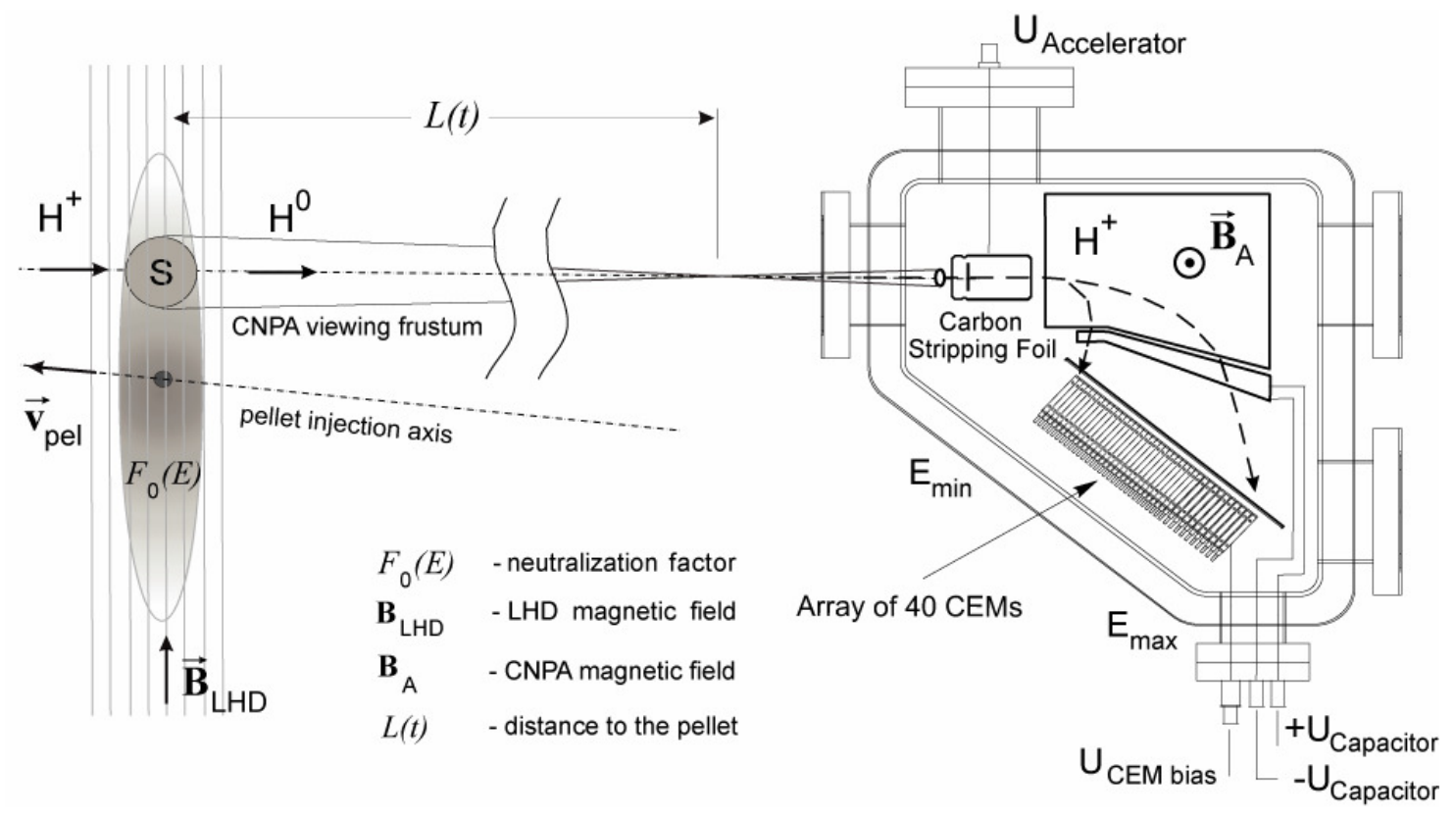

Fig. 1. Local radially resolved measurements of escaping $\mathrm{H}^{0}$ atom energy spectra from LHD plasma column by pellet charge exchange (PCX) method with a compact neutral particle analyzer (CNPA).

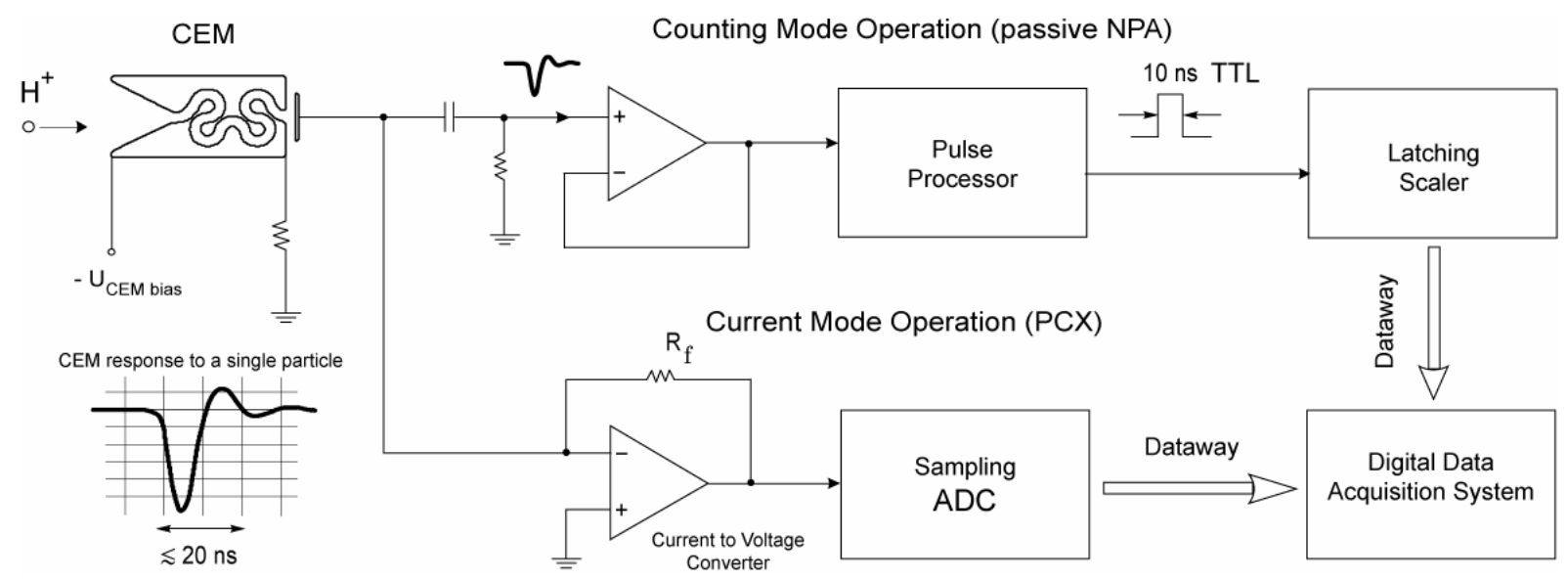

Fig. 2. Particle flux measurement circuit block diagram for one of 40 identical energy channels consisting of a counting mode section for passive NPA (lower fluxes) and a current mode section for PCX measurements (higher particle fluxes typically exceeding $10^{7} \mathrm{~s}^{-1}$ ).

$C \gtrsim N v_{p e l} / \delta l \approx 10^{7} \mathrm{~s}^{-1}$. For such high particle fluxes CEMs can be used in the current mode in addition to the pulse counting mode. CEM signals are fed to two independent sections as shown in Fig.2. Thus, the system is suitable for both passive measurements of naturally occurring neutrals and the active probing with a diagnostic pellet.

\section{Initial Measurement Results}

A clear response of the neutral fluxes to the diagnostic pellet injection has been observed and the energy spectra of PCX neutrals have been obtained with CNPA. The CEM current signals were recorded with $100 \mu$ s time resolution. The measurable energy range was 
$10-100 \mathrm{keV}$ (due to 16 of 40 channels initially available). Ar/He plasma-forming gas was used. The experimental conditions and the obtained energy resolved pellet-induced neutral fluxes are summarized in Fig. 3 illustrating the heating time diagrams and the general discharge waveforms. The left side corresponds to $\mathrm{D}_{p e l}=834 \mu \mathrm{m}, v_{p e l}=385 \mathrm{~m} / \mathrm{s}$ pellet injection in the steady state of $\mathrm{H}$ minority $\mathrm{ICH}$ sustained plasma at $\mathrm{R}_{\mathrm{ax}}=3.6 \mathrm{~m}, \beta=0.09 \%$. The right side corresponds to $\mathrm{D}_{p e l}=867 \mu \mathrm{m}, v_{p e l}=400 \mathrm{~m} / \mathrm{s}$ pellet injection in the steady state of compensated tangential $\mathrm{H}^{0} \mathrm{NBI}$ heated plasma at $\mathrm{R}_{\mathrm{ax}}=3.8 \mathrm{~m}, \beta=0.23 \%$ (unbalanced NBI is likely to cause the pellet trajectory deviation and thus is objectionable for PCX measurements).

The diagnostic pellet injection time corresponds to the spike on the total radiation power curve for each discharge. The millisecond-range pellet ablation time intervals are expanded on the lower two plots showing $\mathrm{H} \alpha$ light intensity from the pellet cloud at $656.3 \mathrm{~nm}$ and the synchronous increase in the CEM current signals reflecting the neutral particle flux. Different curves on the lower plots correspond to different energy channels. Fig. 4 shows the measurement geometry calculation for the ICH heated plasma discharge. This is required to recalculate the time into the effective radius and to interpret the observed particle pitch angles.

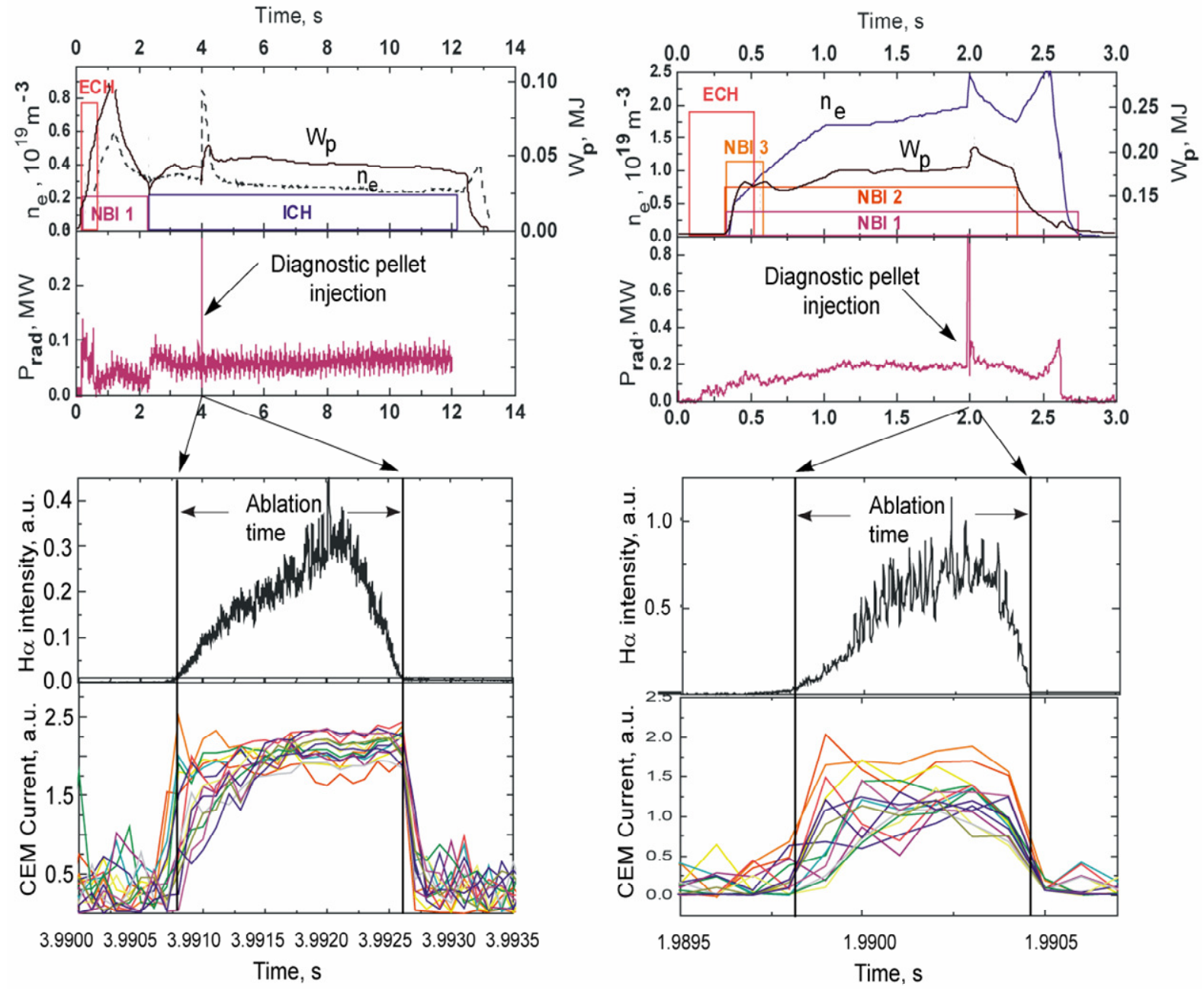

Fig. 3. Energy resolved $\mathrm{H}^{0}$ fluxes synchronous with the visible light from the diagnostic pellet injected into $\mathrm{H}$ minority $\mathrm{ICH}$ sustained $n_{e}=0.4 ? 10^{13} \mathrm{~cm}^{-3} \mathrm{Ar} / \mathrm{He}$ plasma (left) and $\mathrm{H} \mathrm{NBI}$ sustained $n_{e}=2 ? 10^{13} \mathrm{~cm}^{-3} \mathrm{Ar} / \mathrm{He}$ plasma (right). 

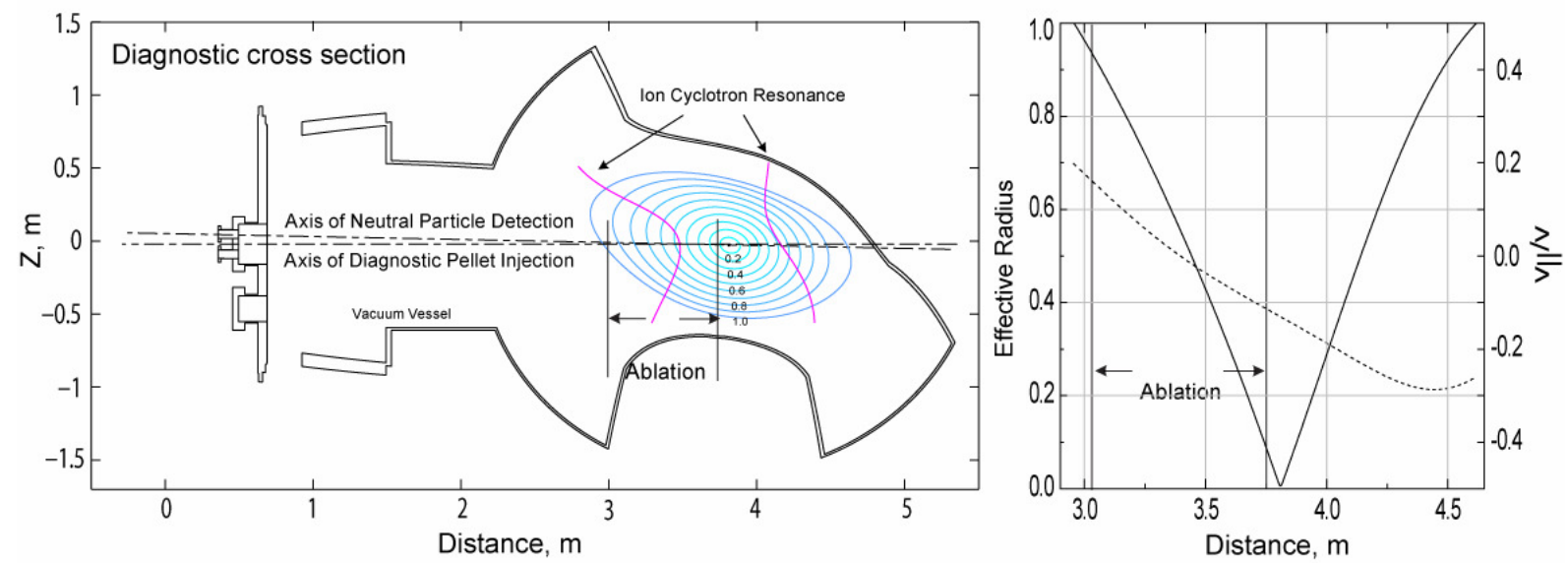

Fig. 4. Range of the radial scan of $\mathrm{ICH}$ plasma by the $\mathrm{D}_{p e l}=834 \mu \mathrm{m}, v_{p e l}=385 \mathrm{~m} / \mathrm{s}$ diagnostic pellet (ablation region) and observable particle pitch angle cosines at $R_{a x}=3.6 \mathrm{~m}, \beta=0.09 \%$.

\section{Data Analysis}

The naturally occurring neutral flux ${ }^{\circ}(E, t)$ depends on the charge exchange target density profile and is calculated as a superposition along the diagnostic sight line expressed by an integral equation with respect to the local neutral source function [5]. The PCX atomic flux ${ }^{P C X}(E, \boldsymbol{r}(t))>>\Gamma^{O}(E, t)$ originates from a localized source and thus one only needs to correct it for all energy-dependent factors to obtain the local ion distribution function.

First, the CEM signals $S(E, t)$ should be corrected for the known detection efficiencies at the corresponding energies since $S(E, t)=\kappa(E){ }^{P C X}(E, \boldsymbol{r}(t))$. The obtained PCX atomic flux itself is expressed as [2]

$$
\Gamma^{(P C X)}(E, \mathbf{r}(t))=\frac{S_{a} S}{4 \pi L^{2}} e^{-\tau(E, L)} F_{0}(E) n_{i}(\mathbf{r}(t)) \mathrm{v}_{i} f_{i}(E, \mathbf{r}(t))
$$

where the geometric factor contains the aperture area $S_{a}$, the visible area of the pellet cloud $S$ and the distance to the cloud L; the exponent accounts for the attenuation of the neutral flux in the plasma on the way out to the periphery; $F_{0}(E)$ is the neutralization factor in the pellet ablation cloud; $f_{i}(E, \boldsymbol{r}(t))$ is the local ion distribution function in the plasma. $F_{0}(E)$ can be calculated from the ionization-recombination balance in the cloud:

$$
F_{0}(E)=\frac{1-\exp \left(-\left(\sigma_{0 \rightarrow 1}(E)+\sigma_{1 \rightarrow 0}(E)\right) \cdot S_{n}\right)}{1+\sigma_{0 \rightarrow 1}(E) / \sigma_{1 \rightarrow 0}(E)} \approx \frac{1}{1+\sigma_{0 \rightarrow 1}(E) / \sigma_{1 \rightarrow 0}(E)}
$$

where $\sigma_{1 \rightarrow 0}$ and $\sigma_{0 \rightarrow 1}$ are hydrogen neutralization and ionization total cross-sections in the cloud respectively and $S_{n}$ is the line-integrated cloud density (across the cloud). If this value is high enough, the approximate formula can be used. Thus, the knowledge of the cloud density and the dominating ion charge states in the cloud during the pellet flight is required as well as the experimental data or theoretical estimations of the relevant charge exchange crosssections. For a polystyrene $\left(-\mathrm{C}_{8} \mathrm{H}_{8}-\right)_{\mathrm{n}}$ pellet the relevant elementary processes are:

$$
\begin{gathered}
H^{+}+C^{k+} \rightarrow H^{0}+C^{(k+1)+}, H^{+}+H^{0} \rightarrow H^{0}+H^{+} \\
H^{0}+C^{k+} \rightarrow H^{+}+C^{(k-1)+}
\end{gathered}
$$



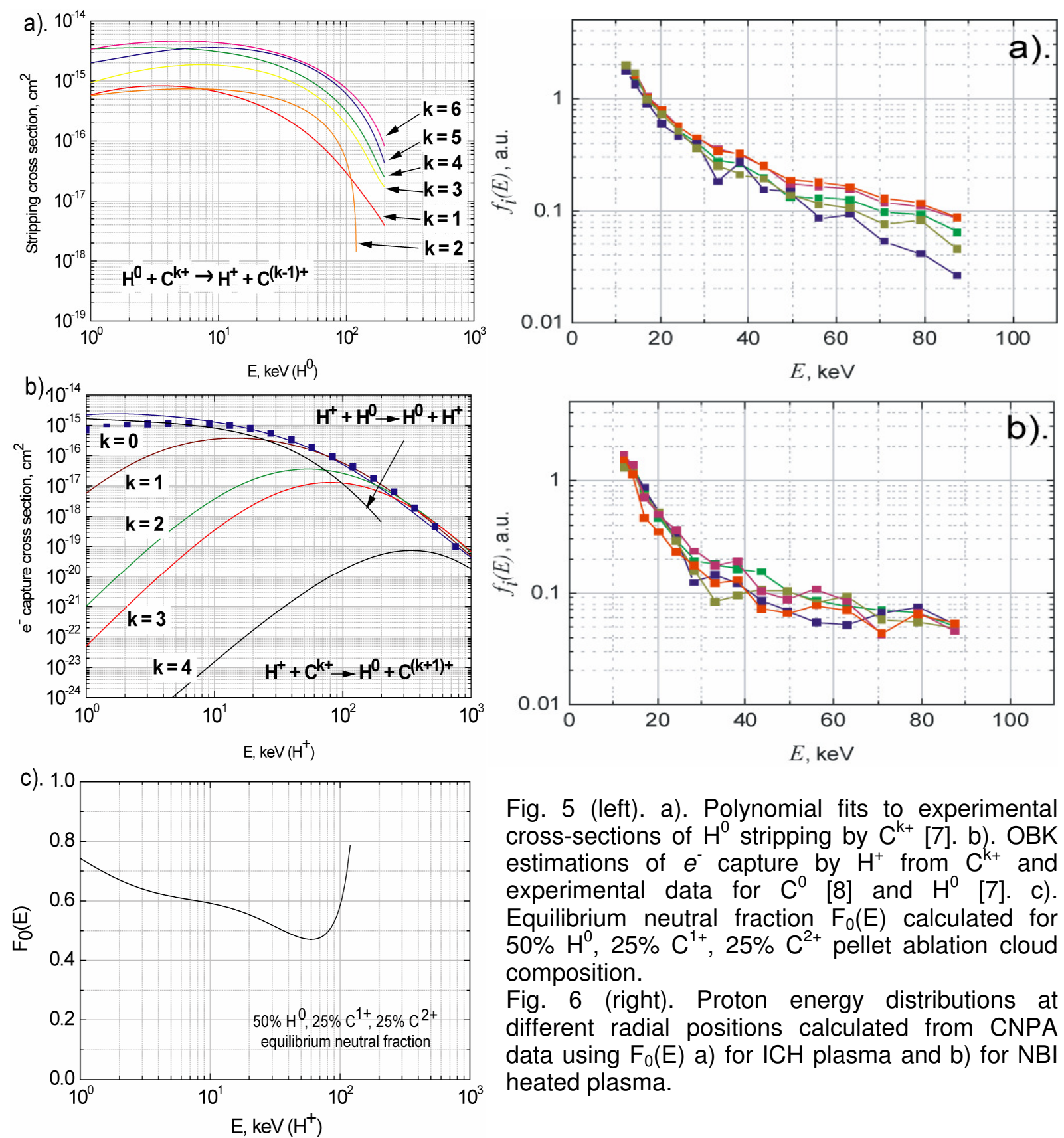

Fig. 5 (left). a). Polynomial fits to experimental cross-sections of $\mathrm{H}^{0}$ stripping by $\mathrm{C}^{k+}[7]$. b). OBK estimations of $e^{-}$capture by $\mathrm{H}^{+}$from $\mathrm{C}^{\mathrm{k}+}$ and experimental data for $\mathrm{C}^{0}[8]$ and $\mathrm{H}^{0}$ [7]. c). Equilibrium neutral fraction $\mathrm{F}_{0}(\mathrm{E})$ calculated for $50 \% \mathrm{H}^{0}, 25 \% \mathrm{C}^{1+}, 25 \% \mathrm{C}^{2+}$ pellet ablation cloud composition.

Fig. 6 (right). Proton energy distributions at different radial positions calculated from CNPA data using $\mathrm{F}_{0}(\mathrm{E})$ a) for $\mathrm{ICH}$ plasma and b) for NBI heated plasma.

The experimental stripping cross-sections for $\mathrm{H}^{0}$ by carbon ions are available [7] as well the very well known hydrogen charge-exchange cross-section. However, the cross-sections of the electron capture by $\mathrm{H}^{+}$from carbon have only been experimentally determined for $\mathrm{C}^{0}[8]$. Therefore, Oppenheimer-Brinkman-Kramers approximation [9] has been used to estimate them. The cross-sections that contribute to $\sigma_{1 \rightarrow 0}$ and $\sigma_{0 \rightarrow 1}$ are shown in Fig.5 a) and b). The ion charge state composition of the pellet cloud has not been experimentally characterized. The assumption made to calculate the neutral fraction is $50 \% \mathrm{H}^{0}, 25 \% \mathrm{C}^{1+}, 25 \% \mathrm{C}^{2+}$. The resultant equilibrium $F_{0}(E)$ is shown in Fig. $5 \mathrm{c}$ ). The PCX atomic flux attenuation was assumed to be negligible.

The obtained $F_{0}(E)$ allows one to calculate the ion distributions from the measured escaping neutral energy spectra. Fig. 6 shows $f_{i}(E)$ at different radial positions. 

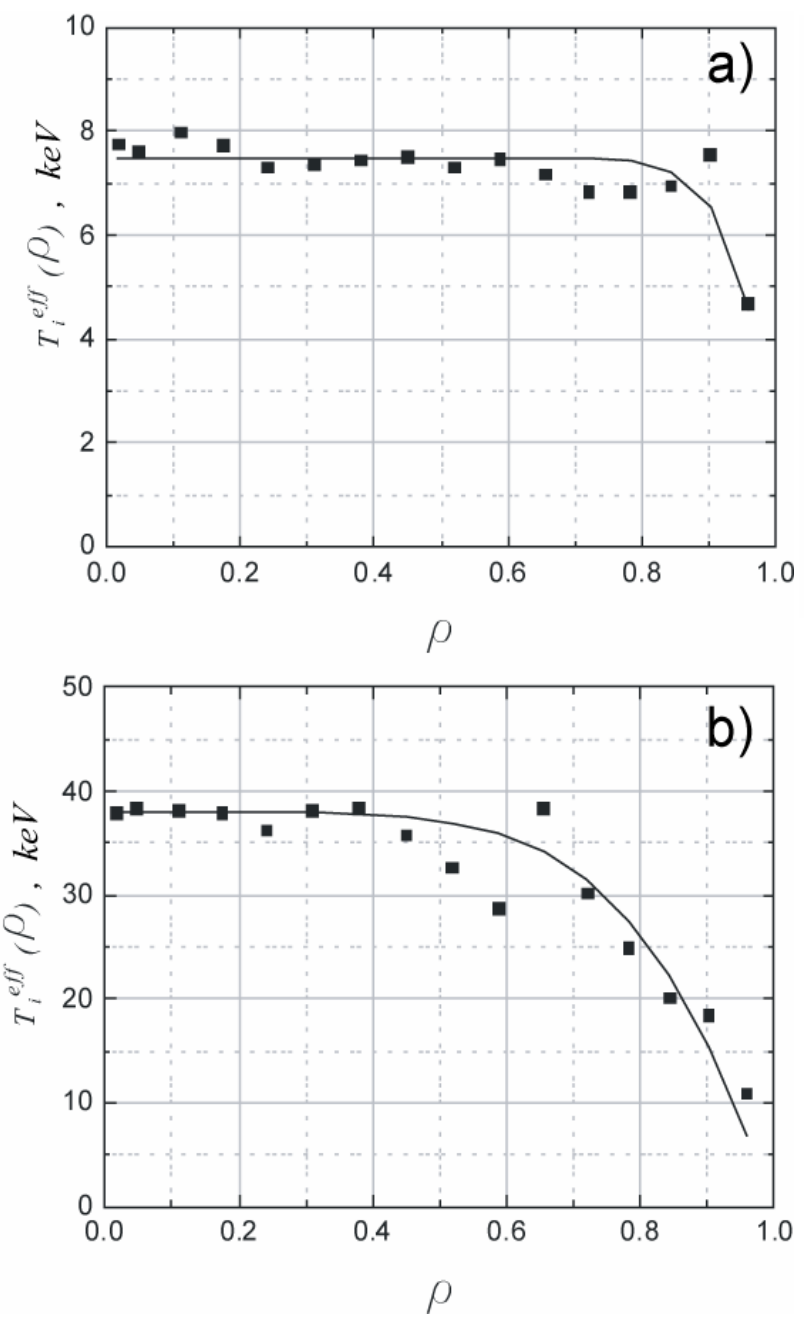

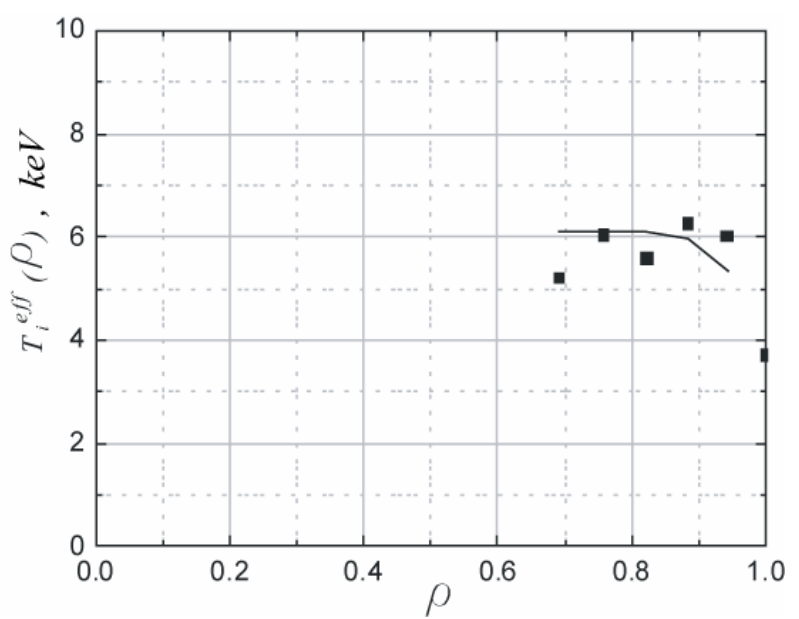

Fig. 7 (left). Radial profiles of effective $\mathrm{H}^{+}$ion temperature in $\mathrm{ICH}$ plasma a). in the lower energy range and b) in the upper energy range.

Fig. 8 (right). Radial dependence of effective $\mathrm{H}^{+}$ion temperature in $\mathrm{NBI}$ heated plasma (lower energy range).

The lower energy distributions below $25 \mathrm{keV}$ are attributed to the thermalized proton population. The high energy tails from tangential NBI heated plasma correspond to the particles that have undergone a significant pitch angle scattering. These tails

at different $\rho$ depend on the NBI source function, i.e. the initial NBI particle angular distribution. In the energy range corresponding to thermalized protons from ICH and NBI and also for high energy tails from ICH an effective temperature is defined as $T_{i}^{\text {eff }}(\rho)=-1 / k(\rho)$ where $k(\rho)$ is the logarithmic slope of $f_{i}(E) / \sqrt{E}$. Fig. 7 shows the effective proton temperature radial profiles calculated from the thermalized particle energy range (a) and also from the high energy tails (b) in ICH plasma. Fig. 8 shows $T_{i}^{e f f}$ radial dependence calculated from the thermalized NBI particle energy range. The simple polynomial fits on all plots have the form $T_{i}^{\text {eff }}(\rho)=T_{i}^{\text {eff }}(0)\left(1-\rho^{\alpha}\right)^{\beta}$ (solid curves).

\section{References}

[1] R.K. Fisher, J.S. Leffler at al., Fusion Technol., 13 (1988), 536

[2] P.R. Goncharov, T. Saida et al., Rev. Sci. Instrum., 74 (2003), 1869

[3] P.R. Goncharov, T. Ozaki et al., Rev. Sci. Instrum., 75 (2004), 3613

[4] F.V. Chernyshev, V.I. Afanasyev et al., Instr. and Exp. Tech., 47 (2004), 214

[5] P.R.Goncharov, J.F. Lyon et al., J. Plasma Fusion Res. Series, 6 (2003), 314

[6] V.Yu.Sergeev et al., $29^{\text {th }}$ EPS Conf. on Contr. Fusion Plasma Phys., vol. 26B, P-2.120 (2002)

[7] C.F. Barnett, ed., Atomic Data for Fusion, ORNL-6086, USA (1990)

[8] P.C. Stancil, J.P. Gu at al., J. Phys. B: At. Mol. Opt. Phys. 31 (1998) 3647

[9] R.A. Mapleton, Theory of Charge Exchange (John Wiley \& Sons, New York, 1972) 